\title{
Effects of ultrasound-guided stellate ganglion block on cervical vascular blood flow: study protocol for a randomized controlled trial
}

\author{
Shaofeng $\mathrm{Pu}^{1}$, Jie Chen ${ }^{2}$, Xing $\mathrm{Gu}^{3}$, Yongming $\mathrm{Xu}^{1}$, Junzhen $\mathrm{Wu}^{1}$, Yingying $\mathrm{Lv}^{1}$ and Dongping $\mathrm{Du}^{1^{*}}$
}

\begin{abstract}
Background: The stellate ganglion block (SGB) can lead to vasodilation of the head and neck. However, controversy remains concerning the changes in extracerebral blood flow. The objective of this study is to assess the effects of SGB on the blood flow to the neck.

Methods: A randomized controlled crossover trial with 38 participants will be conducted. Participants who have primary headaches will be assigned to either group A or B. Patients in group A will receive SGB with $6 \mathrm{ml} 1 \%$ lidocaine, and after a one-week washout period, they will undergo the second SGB with $6 \mathrm{ml}$ normal saline. In contrast, patients in group B will receive the opposite protocol. Data will be collected at baseline (TO) and at 15 min after the first intervention (T1), 15 min before the second intervention (T2), 15 min after the second intervention (T3) and at a 3-week follow up (T4). T1 is the primary time point for the primary outcome analysis. The primary outcomes include the peak systolic velocity (PSV), the end diastolic velocity (EDV), resistance index (RI) and vessel diameter of the common carotid artery (CCA) and vertebral artery (VA). The secondary outcomes include the rate of ptosis, the rate of conjunctival flushing, and the numerical rating scale (NRS) pain score. Additionally, adverse events (AEs) or serious adverse events (SAEs) will be collected at each assessment point.
\end{abstract}

Discussion: This study will comprehensively investigate the efficacy of SGB in extracerebral blood flow. Our research may also suggest that SGB will be effective in reducing pain in patients with primary headaches.

Trial registration: Chinese Clinical Trial Registry, identifier ChiCTR-IOR-17011536. Registered on 1 June 2017.

Keywords: Stellate ganglion block, Blood flow, Primary headaches, Randomized control crossover trial

\section{Background}

The stellate ganglion measures approximately $2.5 \mathrm{~cm}$ in length, $1 \mathrm{~cm}$ in width, and $0.5 \mathrm{~cm}$ in thickness. It is located posteriorly in the chest, in front of the neck of the first rib, and may extend to the seventh cervical (C7) vertebral body [1]. The stellate ganglion block (SGB) is an accepted intervention for the treatment of a variety of pain conditions of the head and neck regions as well as the upper limbs [2]. SGB is also effective in the treatment of

\footnotetext{
* Correspondence: dudp@sjtu.edu.cn

${ }^{1}$ Department of Pain Management, Shanghai Jiao Tong University Affiliated Sixth People's Hospital, 600 Yi Shan Rd, Shanghai 200233, People's Republic of China

Full list of author information is available at the end of the article
}

phantom pain, postherpetic neuralgia, cancer pain, cardiac arrhythmias, orofacial pain, and vascular headache [3].

SGB is conventionally performed using a blind technique. However, this blind technique can cause various adverse effects, such as inadvertent epidural, subarachnoid, or intravascular injection, formation of haematomas, and oesophageal injury [4-6]. Ultrasound-guided SGB was introduced in 1995 [7]. Ultrasound scanning can allow for imaging and distinction of the anatomical structure of the neck. Ultrasound-guided SGB is safer than the conventional technique and allows the use of a small injectate volume while maintaining the same degree of efficacy.

(c) The Author(s). 2018 Open Access This article is distributed under the terms of the Creative Commons Attribution 4.0 International License (http://creativecommons.org/licenses/by/4.0/), which permits unrestricted use, distribution, and reproduction in any medium, provided you give appropriate credit to the original author(s) and the source, provide a link to the Creative Commons license, and indicate if changes were made. The Creative Commons Public Domain Dedication waiver (http://creativecommons.org/publicdomain/zero/1.0/) applies to the data made available in this article, unless otherwise stated. 
SGB leads to vasodilation of the head and neck. However, controversy remains regarding the changes in cerebral and extracerebral blood flow in the head. Liu et al. [8] reported that the blood flow velocities of the internal carotid artery (ICA) on the ischaemic side were decreased and the resistance indexes were increased in 12 patients with ischaemic optic neuropathy (ION) after daily SGB treatment on the affected side with $2-3 \mathrm{~mL}$ of $2 \%$ lidocaine, for a treatment period of 10-15 days. In contrast, the blood flow velocities of the ICA on the ischaemic side were increased and the resistance indexes were decreased. Ohinata et al. [9] reported that patients who underwent SGB experience increased blood flow of the common carotid artery (CCA) and vertebral artery (VA) on the side of the SGB and decreased blood flow on the opposite side. Nitahara et al. [10] showed that SGBs with 6-8 mL of $1 \%$ mepivacaine can significantly increase the blood flow velocity in the CCA, whereas velocity in the VA was unchanged. On the side contralateral to the SGB, significant changes in blood flow velocity in the CCA and VA were never observed. SGB can increase blood flow in the anastomotic artery after superficial temporal artery-middle cerebral artery bypass [11]. In patients with subarachnoid haemorrhage (SAH) after SGB, a significant increase in the calibre of the middle cerebral artery (MCA), VA, and arteriae basilaris (BA) was observed. It has also been shown that the calibre of the MCA, VA, and BA was increased in patients without SAH after treatment with SGB [12].

Kapral et al. [7] and Narouze et al. [13] reported a successful treatment effect with $5 \mathrm{~mL}$ of local anaesthetic in ultrasound-guided SGB. Two studies reported on the optimal volume of local anaesthetic required for successful ultrasound-guided SGB compared to the traditional approach $[14,15]$. One study showed that $4 \mathrm{ml}$ of $0.2 \%$ ropivacaine was sufficient for a successful block [14], while the other showed the optimal volume was $2 \mathrm{ml}$ of $0.5 \%$ mepivacaine [15].

Therefore, there is no strong evidence regarding the effect of SGB on the blood flow of the CCA and VA. The blocking effect may be different due to the different anaesthetic drugs, the different volume, or the number of treatments used in SGB. Our aim was to design and conduct a double-blind, randomized controlled crossover trial to evaluate the effects of ultrasound-guided SGB on the CCA and VA in subjects with primary headaches. We chose to use $6 \mathrm{ml} \mathrm{1 \%}$ lidocaine to perform the SGB because lidocaine is safe and this injection volume can reliably ensure the success of SGB.

\section{Objective}

We designed this prospective, double-blinded, controlled crossover study to investigate the cervical blood flow changes after SGB. The peak systolic velocity (PSV), the end diastolic velocity (EDV), diameter, and resistance index (RI) of the CCA and VA will be measured before and $15 \mathrm{~min}$ after each procedure. The rate of ptosis, conjunctival flushing, and the pain scores will also be recorded.

\section{Trial design}

A double-blinded, randomized controlled crossover trial will be conducted, and all subjects will undergo ultrasound-guided SBG. Group A will first undergo SGB with $6 \mathrm{ml} 1 \%$ lidocaine. After a one-week washout period, this group will receive ultrasound-guided SGB with $6 \mathrm{ml}$ normal saline. In contrast, group B will receive the opposite protocol. That is, group B subjects will first receive SGB with $6 \mathrm{ml}$ normal saline, and after a one-week washout period, this group will receive SGB with $6 \mathrm{ml}$ 1\% lidocaine.

\section{Methods \\ Setting}

This trial is a prospective, investigator and observer-blinded, randomized, crossover trial. This study will be carried out in the Shanghai Jiao Tong University Affiliated Sixth People's Hospital. A flow diagram outlining the trial is provided in Fig. 1. This protocol was designed in accordance with the Standard Protocol Items: Recommendations for Interventional Trials (SPIRIT) guidelines [16], which can be found in Additional file 1 . Adherence reminder meetings will take place before the beginning of the study. Every team member and research physician will be informed of the importance of the trial procedures and flow.

\section{Recruitment}

Recruitment for this study began on 11 June 2017. The trial is currently actively recruiting from Shanghai Jiao Tong University Affiliated Sixth People's Hospital, China. Eligible participants are identified and approached in the pain management centre. The patient's consent to participate in the trial will be obtained prior to any trial-related procedures. Consent will be obtained by an appropriately trained research delegate. Patients will be asked whether they consent to the storage of their contact details so that a qualitative researcher may invite them to participate in a qualitative interview about views on SGB treatment. The qualitative researcher will collect consent for participation in this additional interview immediately prior to commencement of the interview using the qualitative interview Consent Form.

\section{Inclusion criteria}

Participants who meet the diagnostic criteria for primary headaches [17] will be included if they are aged between 18 and 70 years and have signed an informed consent form. 


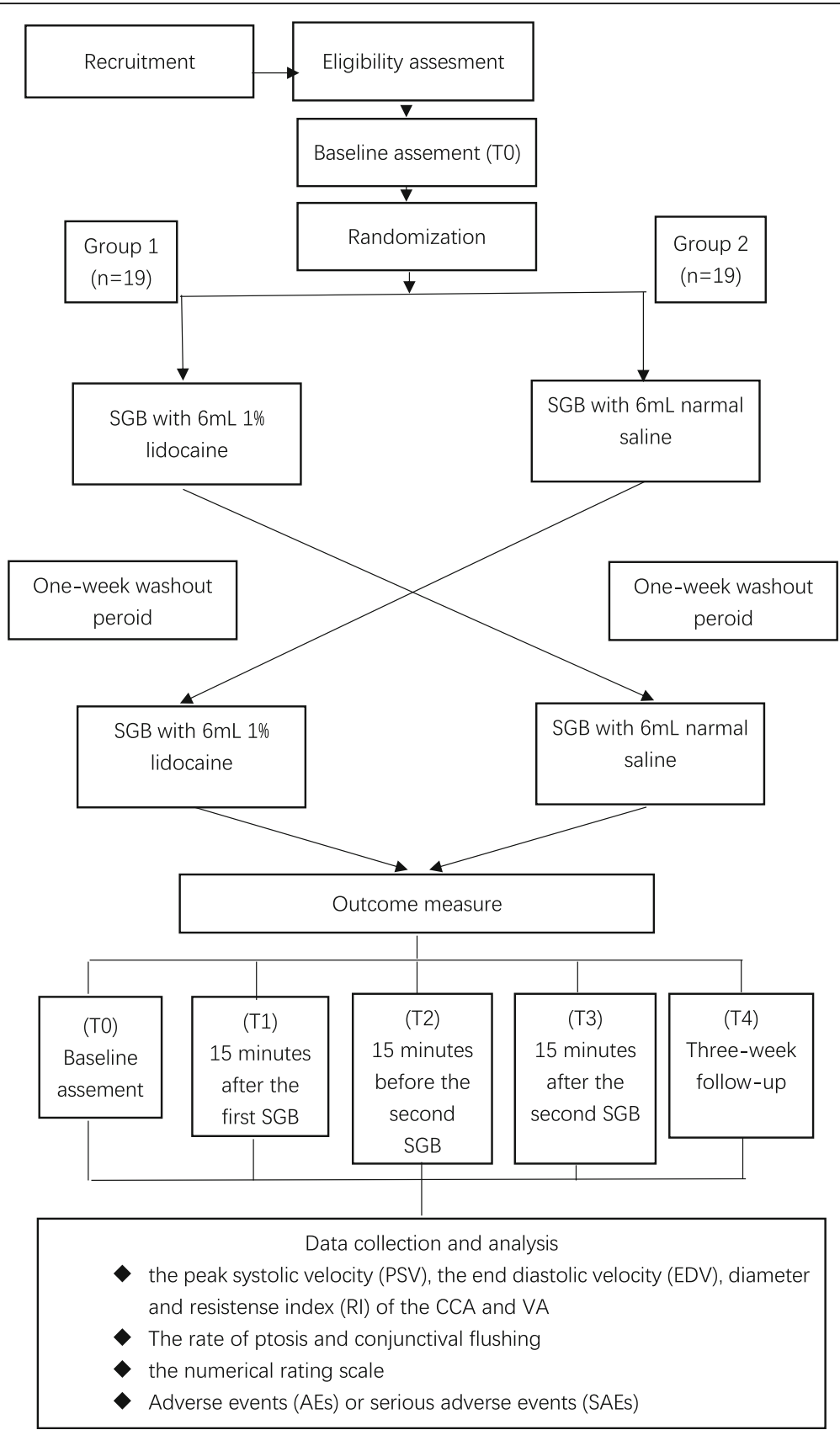

Fig. 1 Participant flow diagram according to Consolidated Standards of Reporting Trials (CONSORT)

\section{Exclusion criteria}

Participants who meet any of the following criteria are not eligible for this study:

1. Participants with a space-occupying lesion

2. Known chronic liver or kidney disease

3. Participants with coagulation disorders

4. Participants with systemic or local infection

5. Participants with drug allergies

6. Psychotic patients

\section{Dropout criteria}

Participants will be withdrawn from the study if they are not willing to continue their participation, cannot be present on the day of the experiment, or miss a treatment session and/or change their form of rehabilitation during the study.

\section{Intervention}

All participants will undergo two SGBs, with a one-week washout period between them. For the ultrasound-guided 
$\mathrm{SGB}$, each patient will receive $6 \mathrm{~mL} 1 \%$ lidocaine and $6 \mathrm{~mL}$ normal saline at one-week intervals to allow a washout period for the injection. The $1 \%$ lidocaine is composed of $2 \%$ lidocaine and normal saline in a 1:1 ratio (for example, the $1 \%$ lidocaine $2 \mathrm{~mL}$ is prepared by combining $2 \%$ lidocaine $1 \mathrm{~mL}$, and normal saline $1 \mathrm{~mL}$ ) $[18,19]$.

The patients will be positioned in a supine position with their neck slightly hyperextended. Prior to the procedure, the $\mathrm{C} 7$ level is confirmed by the use of a $7-14 \mathrm{MHz}$ linear probe (S-Nerve, Sonosite, USA). On the short-axis view, the posterior tubercle and the vertebral body show as a slope in the transverse process of C7. Sonoanatomy of the neck at the $\mathrm{C} 7$ level is confirmed by the absence of the anterior tubercle. The thyroid, inferior thyroid artery, oesophagus, internal jugular vein, CCA, VA, and prevertebral fascia are also confirmed. Colour Doppler imaging is utilised to avoid penetrating the CCA, VA, and the internal jugular vein during the needle insertion. The neck area will be sterilised and the probe will be covered with sterilised vinyl. At the $\mathrm{C} 7$ level, the probe will be placed at the anterior scalene muscle, which is located between the carotid sheath and the brachial plexus. A 25 -gauge, 6 -cm needle will be inserted laterally, $5 \mathrm{~mm}$ from the probe. The needle tip is placed posterior to the carotid artery and anterior to the longus colli muscle under the transverse short axis for the in-plane approach. The assigned dosage of $1 \%$ lidocaine or normal saline will be injected in the patients.

At the end of the SGB, another doctor, who is not involved in the operation, will observe the ptosis and conjunctival flushing. Before and $15 \mathrm{~min}$ after the SGB, the flow measurements will be recorded by an ultrasound specialist. Sonograms will be obtained with a Mylab 90 (Genova, Italy) by using a linear probe (curved-array transducer, CA431, 1-8 MHz).

\section{Expected risks}

The most serious complications of SGB include intravascular injections and retropharyngeal haematoma. The proximity of the stellate ganglion to the inferior thyroid, vertebral, or carotid arteries provides the potential for intravascular injection or vascular trauma, with resulting bleeding and haematoma [20]. Intravascular injection of even small volumes of local anaesthetic may result in loss of consciousness, apnoea, and seizure [21]. Retropharyngeal haematoma varies in severity, from mild and asymptomatic to severe and life-threatening, causing tracheal compression requiring emergency tracheotomy $[22,23]$. The frequency of catastrophic retropharyngeal haematoma after SGB, with resulting airway compromise and obstruction, has been estimated as 1 in 100,000 cases [23]. Compared with the blind technique, however, ultrasound-guided SGB significantly reduced the incidence of asymptomatic haematoma [7].
Ultrasound-guided SGB, with direct visualisation of the multiple vulnerable soft tissue structures enclosed in a tight vascular space around the sympathetic chain, appears to be safer than traditional approaches. To avoid adverse risks to the patients, we will use ultrasound guidance to carefully distinguish the cervical spine, vessels, soft tissue, and trachea. We will also monitor the path and the depth of the needle in real time during ultrasound-guided SGB.

\section{Expected benefits}

Ultrasound-guided SGB may alleviate pain in patients with primary headaches, and it may also contribute to an improved quality of life. Moreover, these participants will play an important role in correctly understanding vascular changes caused by SGB and will contribute to the scientific knowledge on the use of SGB.

\section{Outcomes}

\section{Primary outcomes}

To evaluate the effect of SGB on the vascular blood flow in the neck, the primary outcomes will be the peak systolic velocity (PSV), the end diastolic velocity (EDV), diameter, and resistance index (RI) of the CCA and VA at $15 \mathrm{~min}$ after the first SGB (T1). Sonograms will be obtained with a Mylab 90 (Genova, Italy) by using a $4-10-\mathrm{MHz}$ linear transducer. For flow-volume measurements, a straight segment of the common carotid artery at least $2 \mathrm{~cm}$ below the carotid bulb will be selected [24].

All measurements will be performed by an ultrasound specialist using the same ultrasound instrument.

\section{Secondary outcomes}

Secondary outcomes include: (1) the PSV, EDV, RI, and diameter of the CCA and VA at T0, T2, T3, and T4; (2) the rate of ptosis and conjunctival flushing at $\mathrm{T} 1$ and $\mathrm{T} 3$ (ptosis and conjunctival flushing are considered the standard indicators of SGB of success [25]); (3) the numerical rating scale (NRS) at T0 and T4. The NRS allows the patient to describe the intensity of his/her pain as a number, usually ranging from 0 to 10 , where 0 means no pain and 10 means pain as "bad as it could be" [26].

Adverse events (AEs) or serious adverse events (SAEs) will be collected at each assessment time point and any AEs or SAEs related to the intervention will be reported. For an overview of the schedule of enrolment, interventions, and assessments please see Fig. 2.

\section{Sample size}

The sample size was calculated based on the CCA blood flow results reported previously [11]. With an expected difference of $10 \mathrm{~cm} / \mathrm{s}$ in peak systolic velocity of the CCA between group means, an SD of $10 \mathrm{~cm} / \mathrm{s}, \alpha=0.05$, and $\beta=0.8$, a sample size of 17 patients was required in each group. To compensate for a dropout rate of up to 


\begin{tabular}{|c|c|c|c|c|c|c|}
\hline \multirow[b]{3}{*}{ TIMEPOINT } & \multicolumn{5}{|c|}{ STUDY PERIOD } & \multirow{3}{*}{$\begin{array}{l}\text { Close-out } \\
\text { T4 }\end{array}$} \\
\hline & & \multirow{2}{*}{$\begin{array}{l}\text { Allocation } \\
\text { T0 }\end{array}$} & \multicolumn{3}{|c|}{ Post-allocation } & \\
\hline & $-\mathrm{T} 1$ & & T1 & $\mathrm{T} 2$ & T3 & \\
\hline \multirow{4}{*}{$\begin{array}{l}\text { ENROLMENT: } \\
\text { Eligibility screen } \\
\text { Informed consent } \\
\text { Allocation }\end{array}$} & & & & & & \\
\hline & $x$ & & & & & \\
\hline & $x$ & & & & & \\
\hline & & $x$ & & & & \\
\hline \multicolumn{7}{|l|}{ INTERVENTIONS } \\
\hline \multicolumn{7}{|l|}{ Control } \\
\hline Intervention & & & 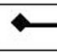 & & $\rightarrow$ & \\
\hline \multicolumn{7}{|l|}{ ASSESSMENTS } \\
\hline \multirow{3}{*}{$\begin{array}{l}\text { Baseline variables } \\
\text { Primary outcomes } \\
\text { Secondary outcomes }\end{array}$} & & $x$ & & & & \\
\hline & & & $x$ & & & \\
\hline & & $x$ & $x$ & $x$ & $x$ & $x$ \\
\hline
\end{tabular}

Fig. 2 Schedule of enrolment, interventions, and assessments. SPIRIT 2013 recommended content for the schedule of enrolment, interventions, and assessments

$10 \%$, we will enrol a total of 38 cases (19 for each group) for this study. Although the study is powered to allow a $15 \%$ dropout rate, we will make every effort to minimise patient loss and missing data during the trial period.

\section{Randomisation}

Participants will be randomly allocated to either group 1 or group 2 with a 1:1 allocation defined by a computer-generated randomisation using the $\mathrm{R}$ package (R Foundation for Statistical Computing). The random allocation sequence will be generated by a statistical expert. Randomisation will be under the control of a single investigator who will be the only person allowed to manage the electronically secured file containing the subject randomisation assignments. This investigator will be blinded to the group to which the participants are allocated.

\section{Blinding}

The participants, researchers, and outcome assessors will remain blind to group allocation throughout the study. Participants will be assigned codes and will be concealed during the allocation process to ensure proper blinding. The researchers responsible for performing the intervention and evaluating the outcomes will not know the study design, allocation, objectives, or expected outcomes.

\section{Data collection process}

We will confirm eligibility criteria are met through review of the participant's medical records. The following data will be collected for each participant: patient identifiers and demographic information, study intervention details, and the peak systolic velocity, vessel diameter, and RI of the CCA and VA, as measured at T0, T1, T2, T3, and T4. The rate of ptosis and conjunctival flushing will be measured at T1 and T3; the pain scores will also be recorded at $\mathrm{T} 0$ and $\mathrm{T} 4$.

\section{Monitoring of data quality}

The peak systolic velocity, vessel diameter, and RI of the CCA and VA will be detected by an ultrasound specialist using the same ultrasound instrument. The rate of ptosis and conjunctival flushing will be recorded by a doctor. Another pain physician will teach patients to use the NRS scoring system and then record pain scores. Data are directly recorded in the electronic study database, which is backed up regularly. All of the assessors will be blinded from the information linking participants and interventions. Study participation will last 3 weeks from enrolment for each participant. No interim analysis will be performed during the study.

\section{Statistical analyses}

Baseline demographic characteristics, including individual variables such as age, sex, weight, and other baseline values, will be expressed with descriptive statistics for the two groups. We will use the SPSS Statistics V. 21.0 $\left(\mathrm{SPSS}_{\mathrm{IBM}}{ }^{\circ}\right)$ to perform all statistical analyses. The normality and homogeneity of all variable distributions will be tested with the Shapiro-Wilk test and Levene's test, respectively. For nonparametric data, the Friedman test will be used followed by the Wilcoxon post hoc test. We will use two-way $(2 \times 4)$ ANOVA for inferential statistical analysis of the parametric data, with Tukey's post hoc 
tests. All data will be represented by the mean \pm standard deviation. Significance level will be set as $\alpha \leq 0.05$.

The main analysis will be performed after all patients have completed the study. A statistical analysis plan will be written before the data are analysed.

\section{Discussion}

The purpose of this study was to investigate the effect of SGB on cervical blood flow and to observe the efficacy of SGB in patients with primary headaches.

Ultrasound techniques are useful both in measurement of the cervical vascular $[27,28]$ and SGB $[6,29,30]$. Cervical sympathetic and SGBs provide a valuable diagnostic and therapeutic benefit to sympathetically maintain pain syndromes in the head. Identifying the correct fascial plane can be achieved with ultrasound guidance, thus facilitating the caudal spread of the injectate to reach the stellate ganglion at $\mathrm{C} 7$ level. This allows for a more effective and precise sympathetic block with the use of a small injectate volume. Accordingly, the risk of vascular and soft tissue injury may be minimised [6].

According to International Classification of Headache Disorders, second edition (ICHD-2)-based studies, primary headaches corresponded to $50.1-78.4 \%$ of headaches and $2.5-23 \%$ of the cases were unclassified [31, 32]. Primary headaches have complex pathophysiologies, and the exact mechanism of the disease is incompletely understood [33]. Therefore, treatment of the primary headaches is also complicated. Blocking the sympathetic nerve aborts an acute attack of cluster headaches and may play a major role in aborting the cluster [34]. We will select patients with primary headache to participate in this study and expect that SGB will alleviate the pain in these patients.

SGB can cause changes in vascular blood flow in the head and neck, but research in this area is limited and often controversial [9-12]. We expect the outcomes of the present study to help us to understand the connections between the cervical sympathetic nervous system and blood vessels and provide additional clinical evidence of the potential benefits of SGB as applied to primary headaches.

\section{Trial status}

Enrolment began on 11 July 2017. As of 12 October 2017, we have enrolled 27 of our target 38 participants in the study. It is expected that recruitment will be completed by December 2017.

\section{Additional file}

Additional file 1: SPIRIT (Standard Protocol Items: Recommendations for Interventional Trials). Completed SPIRIT 2013 checklist of recommended items to address in a clinical trial protocol and related documents. (DOC $123 \mathrm{~kb}$ )

\section{Abbreviations}

AEs: Adverse events; BA: Arteriae basilaris; C7: Seventh cervical;

CCA: Common carotid artery; ChiCTR: Chinese Clinical Trial Registry; CONSORT: Consolidated Standards of Reporting Trials; EDV: End diastolic velocity; ICA: Internal carotid artery; ICHD-2: International Classification of Headache Disorders, second edition; ION: Ischaemic optic neuropathy; MCA: Middle cerebral artery; NRS: Numerical rating scale; PSV: Peak systolic velocity; RI: Resistance index; SAE: Serious adverse event; SAH: Subarachnoid haemorrhage; SGB: Stellate ganglion block; SPIRIT: Standard Protocol Items: Recommendations for Interventional Trials; VA: Vertebral artery

\section{Acknowledgements}

This work was supported through a grant from the National Natural Science Foundation of China. We would like to thank Jiang ZR for his comment on the manuscript. We also thank Zhang JF for critically reviewing the study proposal.

\section{Funding}

This work was supported in part by the General Program of National Natural Science Foundation of China (81370933 and 81672237). The funders will not participate in the study design; collection, management, analysis, and interpretation of data; writing of the report; and the decision to submit the report for publication.

\section{Authors' contributions}

SFP, JC, and DPD conceived and designed the study. XG and SFP drafted the statistical analysis plan. SFP, YMX, and YYL drafted the original protocol. JZW participated in study coordination. All authors have read and approved the final version of the manuscript.

\section{Ethics approval and consent to participate}

This research project was approved by the Ethical Committee of Shanghai Jiao Tong University Affiliated Sixth People's Hospital, China in June 2016 (approval number 2016-44). The study was registered in ChiCTR (Chinese Clinical Trial Registry, ChiCTR-IOR-17011536) on 1 June 2017 (http:// www.chictr.org.cn/index.aspx). Informed consent will be obtained from all potential participants before trial commencement.

\section{Consent for publication}

Not applicable.

\section{Competing interests}

The authors declare that they have no competing interests.

\section{Publisher's Note}

Springer Nature remains neutral with regard to jurisdictional claims in published maps and institutional affiliations.

\section{Author details}

'Department of Pain Management, Shanghai Jiao Tong University Affiliated Sixth People's Hospital, 600 Yi Shan Rd, Shanghai 200233, People's Republic of China. ${ }^{2}$ Department of Ultrasound in Medicine, Shanghai Jiao Tong University Affiliated Sixth People's Hospital, Shanghai Institute of Ultrasound in Medicine, Shanghai 200233, China. ${ }^{3}$ Department of Gynaecology and Obstetrics, the Affiliated Kunshan First People's Hospital, Jiangsu University, Kunshan 215300, China.

Received: 14 October 2017 Accepted: 8 June 2018

Published online: 07 August 2018

References

1. Hogan $\mathrm{QH}$, Erickson SJ. MR imaging of the stellate ganglion: normal appearance. AJR Am J Roentgenol. 1992;158(3):655-9.

2. Aeschbach A, Mekhail NA. Common nerve blocks in chronic pain management. Anesthesiol Clin North Am. 2000;18(2):429-59. viii

3. Elias M. Cervical sympathetic and stellate ganglion blocks. Pain Physician. 2000;3(3):294-304.

4. Shankar H, Simhan S. Transient neuronal injury followed by intravascular injection during an ultrasound guided stellate ganglion block. Anesthesiol Pain Med. 2013;2(3):134-7. 
5. Hirota K, Hirata K, Shibata S, Shigematsu K, Higa K, Yamaura K. Risk vessels of retropharyngeal hematoma during stellate ganglion block. Reg Anesth Pain Med. 2017;42(6):778-81.

6. Narouze S. Ultrasound-guided stellate ganglion block: safety and efficacy. Curr Pain Headache Rep. 2014;18(6):424.

7. Kapral S, Krafft P, Gosch M, Fleischmann D, Weinstabl C. Ultrasound imaging for stellate ganglion block: direct visualization of puncture site and local anesthetic spread. Pilot Study Reg Anesth. 1995;20(4):323-8.

8. Liu F, Xu G, Liu Z, Zhao Y, Lv X, Wang J. The effects of stellate ganglion block on visual evoked potential and blood flow of the ophthalmic and internal carotid arteries in patients with ischemic optic neuropathy. Anesth Analg. 2005;100(4):1193-6.

9. Ohinata Y, Makimoto K, Kawakami M, Haginomori S, Araki M, Takahashi H. Blood flow in common carotid and vertebral arteries in patients with sudden deafness. Ann Otol Rhinol Laryngol. 1997;106(1):27-32.

10. Nitahara K, Dan K. Blood flow velocity changes in carotid and vertebral arteries with stellate ganglion block: measurement by magnetic resonance imaging using a direct bolus tracking method. Reg Anesth Pain Med. 1998;23(6):600-4.

11. Doi S, Cho N, Obara T. Stellate ganglion block increases blood flow in the anastomotic artery after superficial temporal artery-middle cerebral artery bypass. Br J Anaesth. 2016;117(3):395-6.

12. Nie Y, Song R, Chen W, Qin Z, Zhang J, Tang J. Effects of stellate ganglion block on cerebrovascular vasodilation in elderly patients and patients with subarachnoid haemorrhage. Br J Anaesth. 2016;117(1):131-2.

13. Narouze S, Vydyanathan A, Patel N. Ultrasound-guided stellate ganglion block successfully prevented esophageal puncture. Pain Physician. 2007;10(6):747-52.

14. Jung G, Kim BS, Shin KB, Park KB, Kim SY, Song SO. The optimal volume of $0.2 \%$ ropivacaine required for an ultrasound-guided stellate ganglion block. Korean J Anesthesiol. 2011;60(3):179-84.

15. Lee MH, Kim KY, Song JH, Jung HJ, Lim HK, Lee DI, Cha YD. Minimal volume of local anesthetic required for an ultrasound-guided SGB. Pain Med. 2012;13(11):1381-8.

16. Chan AW, Tetzlaff JM, Gotzsche PC, Altman DG, Mann H, Berlin JA, Dickersin K, Hrobjartsson A, Schulz KF, Parulekar WR, et al. SPIRIT 2013 explanation and elaboration: guidance for protocols of clinical trials. BMJ. 2013;346:e7586.

17. Headache Classification Committee of the International Headache Society. The international classification of headache disorders, 3rd edition (beta version). Cephalalgia. 2013;33(9):629-808.

18. Chen YQ, Jin XJ, Liu ZF, Zhu MF. Effects of stellate ganglion block on cardiovascular reaction and heart rate variability in elderly patients during anesthesia induction and endotracheal intubation. J Clin Anesth. 2015;27(2):140-5.

19. Leftheriotis D, Flevari $P$, Kossyvakis C, Katsaras D, Batistaki C, Arvaniti C, Giannopoulos G, Deftereos S, Kostopanagiotou G, Lekakis J. Acute effects of unilateral temporary stellate ganglion block on human atrial electrophysiological properties and atrial fibrillation inducibility. Heart Rhythm. 2016;13(11):2111-7.

20. Narouze S. Beware of the "serpentine" inferior thyroid artery while performing stellate ganglion block. Anesth Analg. 2009;109(1):289-90.

21. Mahli A, Coskun D, Akcali DT. Aetiology of convulsions due to stellate ganglion block: a review and report of two cases. Eur J Anaesthesiol. 2002;19(5):376-80.

22. Higa K, Hirata K, Hirota K, Nitahara K, Shono S. Retropharyngeal hematoma after stellate ganglion block: analysis of 27 patients reported in the literature. Anesthesiology. 2006;105(6):1238-45. discussion 1235A-1236A

23. Okuda Y, Urabe K, Kitajima T. Retropharyngeal or cervicomediastinal haematomas following stellate ganglion block. Eur J Anaesthesiol. 2003;20(9):757-9.

24. Oktar SO, Yucel C, Karaosmanoglu D, Akkan K, Ozdemir H, Tokgoz N, Tali T. Blood-flow volume quantification in internal carotid and vertebral arteries: comparison of 3 different ultrasound techniques with phase-contrast MR imaging. AJNR Am J Neuroradiol. 2006;27(2):363-9.

25. van Gastel P, Kallewaard JW, van der Zanden M, de Boer H. Stellateganglion block as a treatment for severe postmenopausal flushing. Climacteric. 2013;16(1):41-7.

26. Lee JJ, Lee MK, Kim JE, Kim HZ, Park SH, Tae JH, Choi SS. Pain relief scale is more highly correlated with numerical rating scale than with visual analogue scale in chronic pain patients. Pain Physician. 2015;18(2):E195-200.
27. Yuan SM. Aberrant origin of vertebral artery and its clinical implications. Braz J Cardiovasc Surg. 2016:31(1):52-9.

28. Kalfaoglu ME, Hizal M, Kiyan A, Gurel K. The effects of chronic smoking on total cerebral blood volume measured by carotid and vertebral artery doppler ultrasonography. J Clin Ultrasound. 2017;45(9):561-65.

29. Narouze S, Souzdalnitski D. Ultrasound-guided percutaneous cervical and upper thoracic sympathetic chain neuromodulation for upper extremity complex regional pain syndrome. Ochsner J. 2017;17(2):199-203.

30. Imani F, Hemati K, Rahimzadeh P, Kazemi MR, Hejazian K. Effectiveness of stellate ganglion block under Fuoroscopy or ultrasound guidance in upper extremity CRPS. J Clin Diagn Res. 2016;10(1):UC09-12.

31. Guerrero AL, Rojo E, Herrero S, Neri MJ, Bautista L, Penas ML, Cortijo E, Mulero P, Fernandez R. Characteristics of the first 1000 headaches in an outpatient headache clinic registry. Headache. 2011;51(2):226-31.

32. Pedraza MI, Mulero P, Ruiz M, de la Cruz C, Herrero S, Guerrero AL. Characteristics of the first 2,000 patients registered in a specialist headache clinic. Neurologia. 2015;30(4):208-13.

33. Buture A, Gooriah R, Nimeri R, Ahmed F. Current understanding on pain mechanism in migraine and cluster headache. Anesthesiol Pain Med. 2016;6(3):e35190.

34. Albertyn J, Barry R, Odendaal CL. Cluster headache and the sympathetic nerve. Headache. 2004:44(2):183-5.

\section{Ready to submit your research? Choose BMC and benefit from:}

- fast, convenient online submission

- thorough peer review by experienced researchers in your field

- rapid publication on acceptance

- support for research data, including large and complex data types

- gold Open Access which fosters wider collaboration and increased citations

- maximum visibility for your research: over $100 \mathrm{M}$ website views per year

At BMC, research is always in progress.

Learn more biomedcentral.com/submissions 